\title{
ESSAI \\ DE PRODUCTIONS AQUICOLES INTEGREES : POSSIBILITES D'UTILISATION DES BOUES DE PISCICULTURE COMME AMENDEMENT ORGANIQUE AGRICOLE
}

\author{
R. LESEL, C. JUSTE * et J. KOENIG ** \\ avec la collaboration technique de P. SOLDA * \\ INRA - Centre de Recherches hydrobiologiques \\ Laboratoire des Microorganismes, B.P. 79, \\ 64200 Biarritz
}

\section{RESUME}

Des essais comparatifs ont été faits dans le but de déterminer l'efficacité des boues de pisciculture comme amendement organique. II apparait que ces boues constituent un amendement aussi efficace qu'un poids équivalent de fumier de ferme.

\section{ABSTRACT}

An attempt at integrated aquacultural productions: possibilities in the utilization of hatchery deposits as an agricultural organic fertilizer.

Comparative tests have been carried out in order to determine the efficiency of hatchery deposits as an organic fertilizer. It is shown that these deposits constitue a fertilizer as efficient as farm manure of an equivalent weight.

(*) INRA, Station d'Agronomie * La Grande Ferrade *, 33140 Pont-de-la-Maye.

$\left({ }^{* *}\right)$ SARB-CLEN, rue Wilson, B.P. 48,69150 Decines. 


\section{I. - INTRODUCTION}

Nous avons montré dans une étude prëcédente (LESEL et IFERGAN, 1975) qu'il est possible d'utiliser la charge azotée d'une eau usée de pisciculture pour assurer la croissance du cresson. Nasturtium officinale. Rappelons que le principe directeur de ces travaux consiste à organiser une chaine trophique artificielle où des consommateurs à divers niveaux récupèrent et transforment toute l'énergie apportée au système essentiellement sous forme d'aliments. Un tel système a été proposé par CLARKE (1972). Le schéma général peut varier considérablement. Nous nous sommes proposés d'analyser l'étape 4 du modèle suivant.

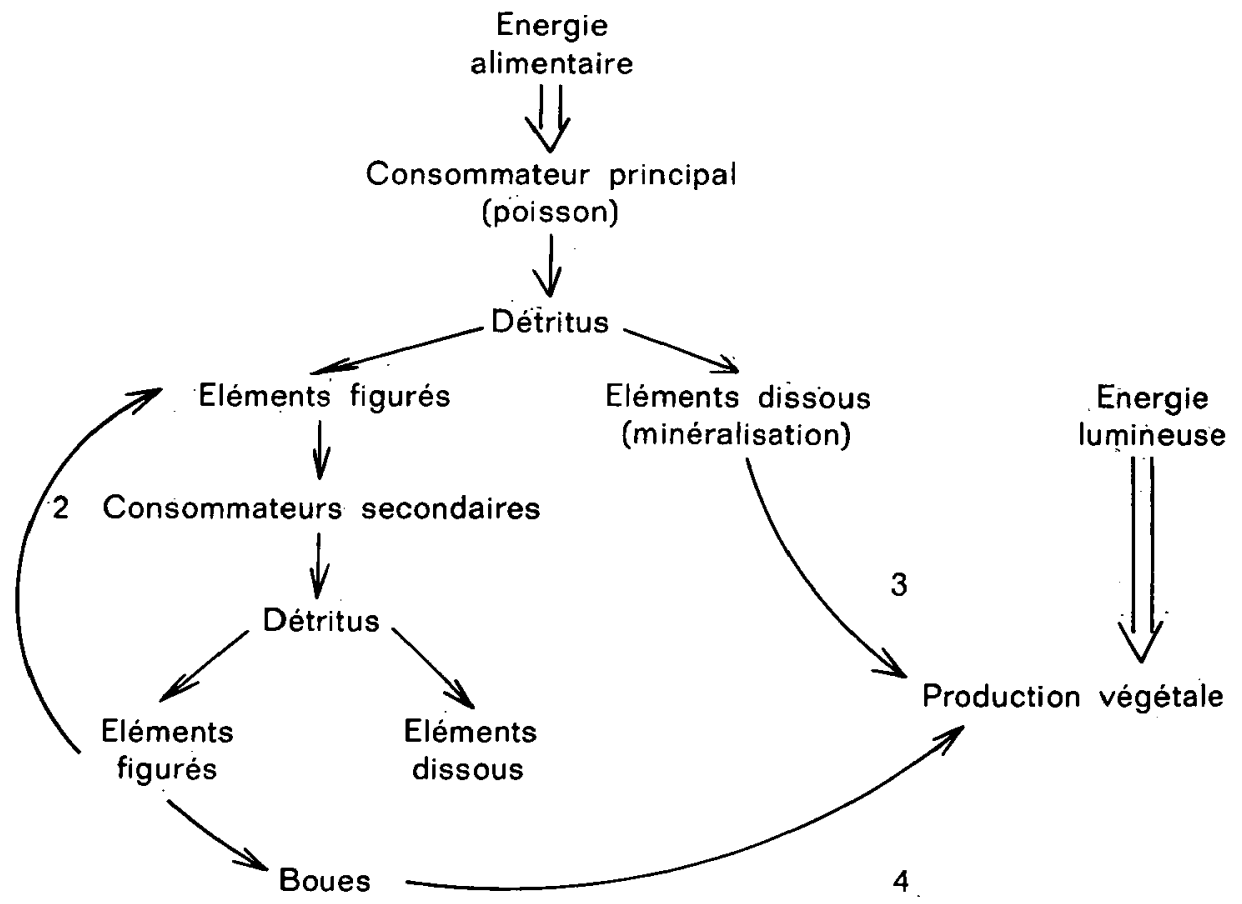

II s'agissait d'utiliser les boues provenant d'élevages de poissons. Ces boues sont d'origines diverses : fèces, refus alimentaires, floculation de substances en solution, et sont en partie responsables de la pollution organique observée en aval des piscicultures. L'étude entreprise avait pour objet la comparaison de l'efficacité de ces boues comme amendement organique avec celle du fumier de ferme.

\section{2. - ETUDE EXPERIMENTALE}

\section{1. - Origine des boues}

Les boues utilisées proviennent de la pisciculture de Font-Rome * à Manthes dans la Drôme. Les prélèvements ont été effectués en avril 1975. A cette époque le taux de recirculation des eaux était de $50 \mathrm{p}$. 100. Les caractéristiques

(*) Les auteurs remercient $M$. BEAL qui leur a aimablement communiqué les renseignements concernant sa pisciculture et fourni la boue utilisée lors des expérimentationis. 
physiques et chimiques de l'eau à l'entrée et à la sortie de la pisciculture montrent qu'il s'agit d'une eau calcaire relativement riche en nitrates (tabl. 1). Les résultats proviennent d'une analyse effectuée en octobre 1974.

\begin{tabular}{|c|c|c|}
\hline $\begin{array}{l}\text { Caractéristiques } \\
\text { physiques }\end{array}$ & $\begin{array}{l}\text { Eau entrée } \\
\text { pisciculture }\end{array}$ & $\begin{array}{l}\text { Eau sortie } \\
\text { pisciculture }\end{array}$ \\
\hline $\begin{array}{l}\text { Aspect } \ldots \ldots \ldots \ldots \ldots \ldots \\
\text { Odeur } \ldots \ldots \ldots \ldots \ldots \ldots \ldots \\
\text { Température } \ldots \ldots \ldots \ldots \ldots \\
\text { pH (laboratoire) } \ldots \ldots \ldots \ldots \ldots \\
\text { Conductivité en mhos à } 20^{\circ} \mathrm{C} \\
\text { Matières totales } \\
\quad \text { en suspension } \ldots \ldots \ldots \ldots \ldots\end{array}$ & $\begin{array}{c}\text { claire, fin dépôt } \\
\text { néant } \\
1005 \\
7,2 \\
505 \\
2,8\end{array}$ & $\begin{array}{c}\text { claire } \\
\text { néant } \\
1105 \\
7,5 \\
497 \\
<0,5\end{array}$ \\
\hline $\begin{array}{l}\text { Caractéristiques } \\
\text { chimiques }\end{array}$ & $\begin{array}{l}\text { Eau entrée } \\
\text { pisciculture }\end{array}$ & $\begin{array}{l}\text { Eau sortie } \\
\text { pisciculture }\end{array}$ \\
\hline 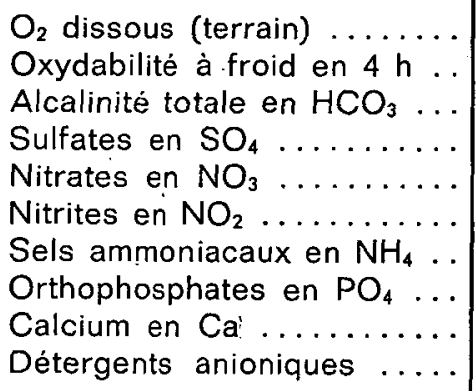 & $\begin{array}{l}11 \\
0,45 \\
295 \\
\text { présence } \\
27 \\
0 \\
0 \\
0 \\
126 \\
0\end{array}$ & $\begin{array}{l}8,0 \\
0,60 \\
275 \\
\text { présence } \\
29 \\
0,06 \\
0 \\
-0 \\
120 \\
0\end{array}$ \\
\hline
\end{tabular}

Tableau 1. - Caractéristiques physiques et chimiques des eaux de pisciculture (analyses effectuẻes par le Laboratoire du CTGREF, Division Qualité des Eaux, Pêche et Pisciculture, Paris).

Table 1. - Physical and chemical characteristics of hatchery waters (analyses worked out by the CTGREF Laboratory (Centre Technique du Génie Rural, des Eaux et des Forêts), Division of Water Quality, Fishing and Fish Farming, Paris. 


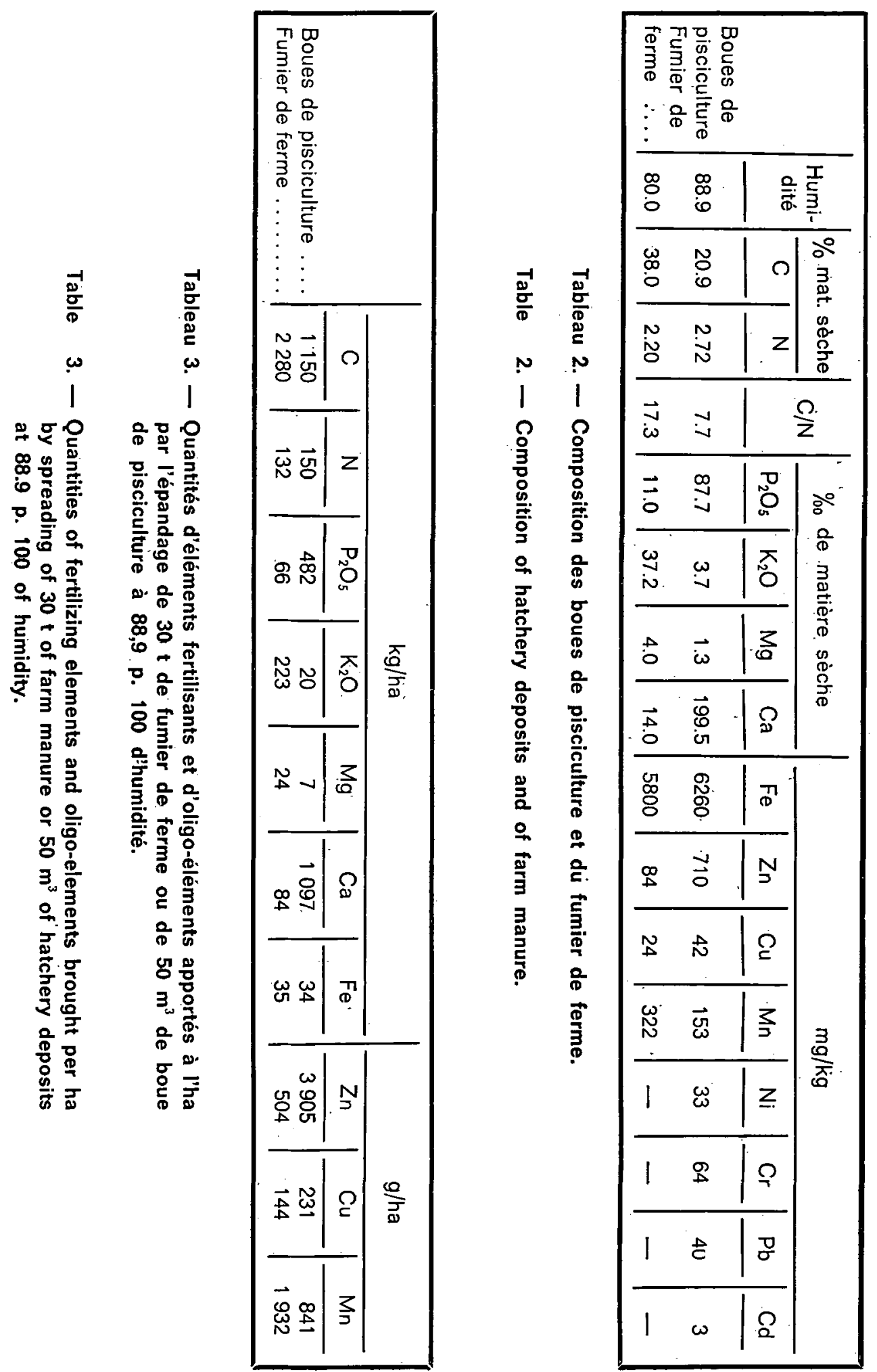




\section{2. - Aspect et composition des boues de pisciculture}

Le produit se présente sous forme d'une suspension en milieu liquide titrant $110 \mathrm{~g}$ environ de matière sèche par litre. Après séchage à l'air, la boue est pulvérisée par passage dans un broyeur. Les dosages sont effectués sur la poudre ainsi obtenue et les résultats ramenés à la matière sèche. Le carbone et l'azote total sont déterminés en utilisant les méthodes de ANNE et de KJELDAHL. Pour les autres éléments, on procède à une calcination au four à $450^{\circ} \mathrm{C}$ pendant $8 \mathrm{~h}$. Les cendres sont reprises par de l'acide chlorhydrique pur et on mesure la teneur en acide phosphorique par réduction du complexe phosphomolybdique réduit par l'acide ascorbique, les métaux étant dosés directement par absorption atomique.

Les résultats qui figurent dans le tableau 2, dans lequel on a fait mention également à titre de comparaison de la composition du fumier de ferme utilisé pour l'essai, montrent que le produit possède une teneur en azote aussi importante que celle du fumier, un rapport $\mathrm{C} / \mathrm{N}$ faible, une concentration très élevée en acide phosphorique et calcium ; par contre, les taúx de potasse et de magnésie sont beaucoup plus faibles que ceux du fumier. Ce tableau fait ressortir en outre, par rapport au fumier, une charge non négligeable en oligo-éléments et métaux lourds, notamment en fer, zinc et manganèse. Ces oligo-éléments peuvent provenir des farines animales entrant dans la composition du poisson ou, plus simplement, d'une concentration des éléments-traces présents dans les eaux, cette accumulation des métaux lourds par les boues ètant un phénomène bien connu. II convient cependant de préciser que les teneurs mesurées dans ce type de boues sont beaucoup plus faibles que celles que l'on rencontre habituellement dans des boues issues par exemple du traitement des eaux usées urbaines.

Pour mieux fixer les idées, on a indiqué dans le tableau 3 les quantités des différents éléments qui seraient apportés à l'hectare de sol cultivé sur lequel on aurait effectué un arrosage de $5 \mathrm{~mm}$ à l'aide des boues de pisciculture à l'état frais. On a représenté. également dans ce tableau les quantités d'éléments qui seraient apportées par l'épandage de 30 t/ha de fumier de ferme à 80 p. $100 \mathrm{~d}$ 'humidité, dose couramment employée en agriculture.

Ce tableau met en évidence que l'épandage de $50 \mathrm{~m}^{3} / \mathrm{ha}$ de boues de pisciculture correspond à un apport d'éléments fertilisants non négligeable, notamment en ce qui concerne l'azote et surtout l'acide phosphorique, alors que les quantités de potasse incorporées de cette matière sont beaucoup plus faibles, voire négligeables quand on les compare à celles qu'amène le fumier. Outre les éléments majeurs, les boues amènent au sol des quantités importantes d'oligoéléments, particulièrement. de zinc et de fer.

La simple analyse des boues de pisciculture fait donc apparaitre un certain potentiel en tant que source d'éléments nutritifs; encore faut-il s'assurer du caractère non-phytotoxique de ce matériau et essayer de mettre en évidence un effet bénéfique éventuel, lié à autre chose qu'une aptitude à fonctionner comme source d'éléments nutritifs pour la plante. Pour cela, on a réalisé en serre un essai en vases de végètation portant une culture-test, en l'occurrence du raygrass d'ltalie, dont on a comparé le comportement : d'une part, en présence de fumier de ferme (l'analyse de ce dernier figure dans le tableau 2), d'autre part, de boues de pisciculture, les deux amendements étant introduits dans le substrat de culture en quantités identiques. 


\section{3. - Dispositif expérimental utilisé}

L'essai a été conduit en petits vases de végétation en matière plastique; dans lesquels on a introduit $1,4 \mathrm{~kg}$ de sable lavé à l'acide et débarrassé de toute impureté organique.

Tous les pots reçoivent une fertilisation minérale identique, comportant:

- de l'azote 'ammonitrate 33 p. 100),

- de l'acide phosphorique (superphosphate 25 p. 100),

- de la potasse (sulfate de potasse).

- de la magnésie (sulfate de magnésium).

Cette fertilisation. équivaut à l'épandage de $150 \mathrm{u} / \mathrm{ha}$ de chacun de ces éléments majeurs.

Les pots reçoivent également au cours de leur remplissage :

- $1 \mathrm{~kg}$ de carbonate de calcium,

- $1 \mathrm{~kg}$ de. sulfate de calcium,

de manière à uniformiser les $\mathrm{pH}$ et à pallier tout risque de carence en soufre. On sème dans chaque pot $1 \mathrm{~g}$ de ray-grass d'Italie, et on maintient l'humídité du substrat à 75 p. 100 de la capacité de rétention en eau par des arrosages quotidiens à l'eau permutée. Quatre coupes espacées de trois à quatre semaines ont été réalisées. A partir de la première coupe et tous les 15 jours, les pots reçoivent un complément de fertilisation minérale apportée sous forme de solution nutritive préparée à partir des sels suivants : nitrate d'ammonium, phosphate monopotassique, sulfate de potassium et sulfate de magnésium; la quantité de solution nutritive ajoutée à chaque arrosage $(25 \mathrm{ml})$ équivaut à un épandage de $150 \mathrm{u} / \mathrm{ha}$ d'azote, d'acide phosphorique et de potasse, et de $75 \mathrm{u} / \mathrm{ha}$ de magnésie.

Le ray-grass se trouve donc placé ainsi dans des conditions de nutrition minérale optimales. Les différences de végétation qui peuvent apparaitre à la suite de l'introduction du fumier ou des boues de pisciculture (ajoutés sous forme de poudre et intimement mélangés au sable) traduisent donc: soit un effet phytotoxique de l'amendement expérimenté, soit - dans le cas d'une stimulation de la croissance - une action lièe à autre chose que l'aptitude de cet amendement à fournir des éléments nutritifs.

\section{4. - Protocole expérimental}

L'essai comporte cinq traitements, répétés chacun cinq fois :

$1=$ témoin sans apport de matière organique ;

2 = apport d'une quantité de boue de pisciculture correspondant à 9 t/ha de matière sèche ;

3 = apport d'une quantité de fumier de ferme correspondant à $9 \mathrm{t} / \mathrm{ha}$ de matière sèche ;

$4=$ fertilisation minérale supplémentaire introduite au moment du remplissage des pots, correspondant à la quantité d'éléments fertilisants présents dans les neuf tonnes de boues de pisciculture ;

$5=$ fertilisation minérale supplémentaire introduite au moment du remplissage des pots, correspondant à la quantité d'éléments fertilisants présents däns les neuf tonnes de fumier. 


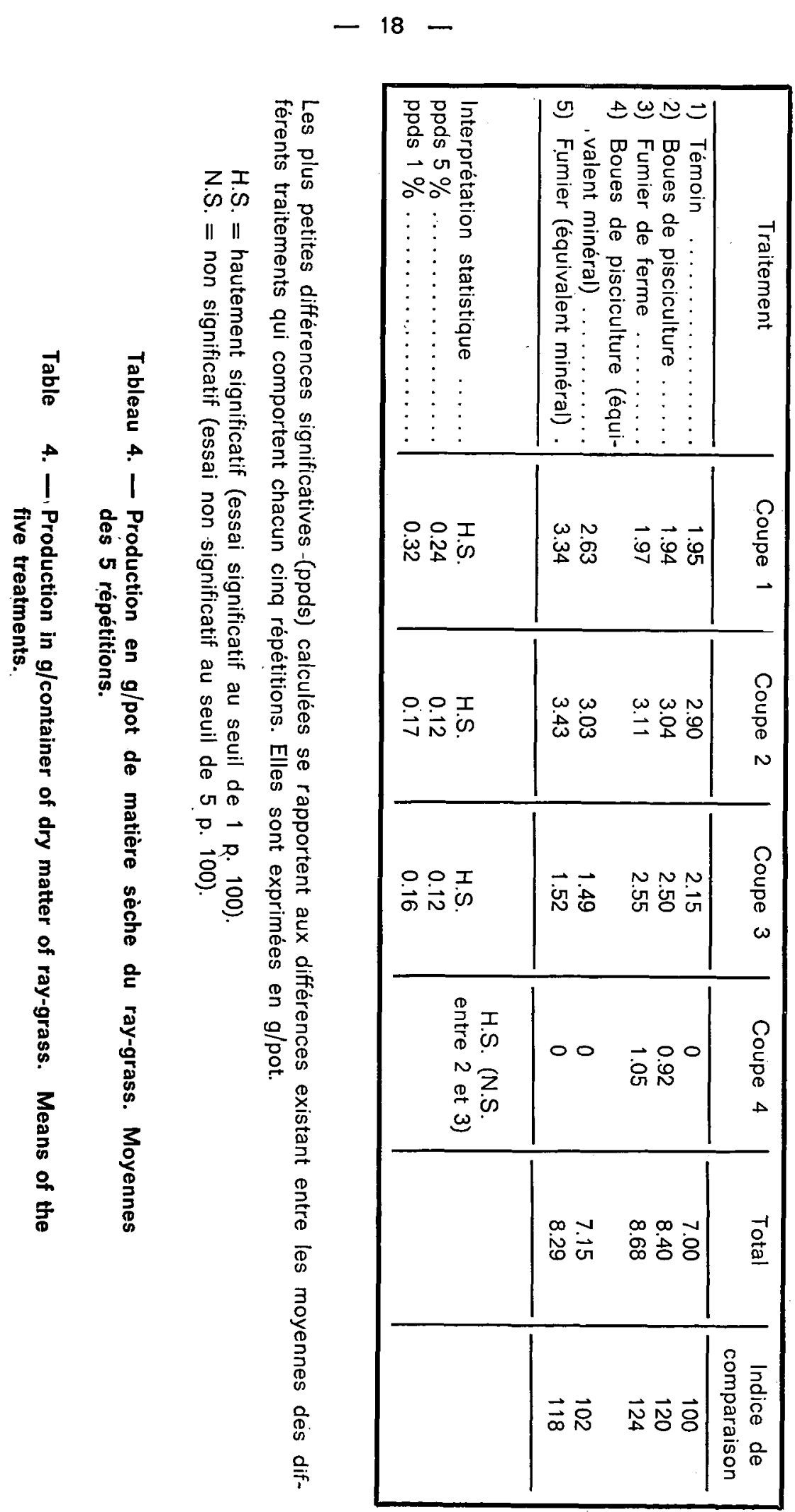


Les différences entre les traitements 2 et 4 d'une part, et 3 et 5 d'autre part, permettent de mieux préciser un effet spécifique éventuel des amendements mis en comparaison, qui ne soit pas lié à leur teneur en éléments fertilisants.

Les déterminations effectuées ont consisté à déterminer la production de matière sèche de chaque pot.

\section{5. - Résultats obtenus (tabl. 4)}

A la première coupe, les pots qui ont reçu l'équivalent minéral de. 9 t/ha de boues ou de fumier fournissent une production nettement supérieure à celle obtenue en présence des amendements organiques: cette différence s'explique sans doute par le fait que les éléments nutritifs présents dans les amendements, et surtout l'azote, sont, progressivement libérés.

A la coupe suivante, en effet, ce phénomène s'atténue, puis s'inverse totalement à partir de la troisième coupe. A la quatrième coupe, les pots qui n'ont pas reçu d'amendement organique ne fournissent aucune récolte. Cette absence de végétation peut s'expliquer, soit par une déficience en un ou plusieurs oligoéléments, soit par l'accumulation dans le milieu jusqu'à des doses toxiques d'éléments nutritifs imparfaitement utilisés par la plante en l'absence de matière organique.

On peut observer que si, pour l'ensemble des 4 coupes, les matières organiques conduisent à un supplément de récolte de l'ordre de 20 p. 100, il n'apparait pas au cours de toute la culture de différence significative entre les deux types de composts organiques expérimentés.

On peut donc conclure que, dans les coriditions expérimentales mises en jeu, un poids donné de boue de pisciculture s'avère être un amendement organique aussi efficace que le même poids de fumier.

\section{3. - CONCLUSIONS}

Cette expérience montre qu'il est possible d'employer les substances organiques et minérales figurées rejetées par une pisciculture utilisant un recyclage même partiel des eaux. TENORE, GOLDMAN et CLARNER (1973) et TENORE, MASON et CHESNAY (1974) ont entrepris la valorisation de ces déchets par des groupes zoologiques détritiphages. Dans une première étape, l'utilisation comme amendement organique des țoues de pisciculture constitue une approche plus simple de cette valorisation. Les deux méthodes peuvent être complémentaires, aboutissant à une meilleure utilisation du flux énergétique distribué sous forme d'aliments au consommateur principal, le poisson.

\section{4. - BibliographiE}

CLARKE R., 1972. Pour un progrès en douceur. Sci. Av., 308, 870-877.

LESEL R., IFERGAN Catherine, 1975. Essai de productions aquicoles intégrées : utilisation des eaux de rejets d'un circuit fermé expérimental de pisciculture pour la culture de cresson. Bull. Fr. Piscic., 259, 41-52.

TENORE K:R., GOLDMAN J.S., CLARNER J.P., 1973. The food chain dynamics of the oyster, clam and musse! in an aquaculture food chain. J. exp. mar. Biol. Ecol., 12, 157-165.

TENORE K.R., MASON.G.B., CHESNAY E.J. Jr., 1974. Polyspecies aquaculture systems : the detrital trophic level, J. mar. Res., 32 (3), 425-432. 